\title{
A Fluorescent Antenna for White Light
}

\section{Visible Light Communications}

\author{
Amna Riaz \\ Department of Engineering Sceinces \\ University of Oxford \\ Oxford, United Kingdom \\ amna.riaz@eng.ox.ac.uk
}

\author{
Grahame Faulkner \\ Department of Engineering Sceinces \\ University of Oxford \\ Oxford, United Kingdom \\ grahame.faulkner@eng.ox.ac.uk
}

\author{
Steve Collins \\ Department of Engineering Sceinces \\ University of Oxford \\ Oxford, United Kingdom \\ steve.collins@eng.ox.ac.uk
}

\begin{abstract}
Light emitting diodes are an important pillar of modern lighting systems that could potentially be used to transmit data and hence provide communications channels to augment WiFi. However, lighting LEDs have a limited bandwidth and so high signal to noise ratios (SNRs) are required if they are to transmit an acceptable data rate. Conventional optical concentrators could be used to increase the SNR at the receiver but they limit its field of view. In contrast, fluorescent concentrators can increase the receiver signal whilst preserving a wide field of view. In this paper, results are reported of experiments using a white LED as a transmitter and a receiver that includes a commercially available fluorescent fibre. Data rates at a bit error rate of $10^{-3}$ are reported and a method of relating gain and $\mathrm{OOK}$ data rate is proposed.
\end{abstract}

Keywords-WLED, OOK, fluorescent concentrators, luminescent solar concentrators, Gain, BER

\section{INTRODUCTION}

The popularity of Wi-Fi means that these systems are running out of local capacity and interference between users means that the quality of service can be degraded [1]. For example, Huawei have estimated that when an access point with a capacity of $900 \mathrm{Mbps}$ is shared by 18 users each user receives $6 \mathrm{Mbps}$ and just over $10 \%$ of the available capacity is used. The solution that they have adopted to solve this problem is to add another RF channel [2]. However, the RF spectrum is regulated and so other parts of the electromagnetic spectrum are being considered as sources of additional local capacity [3]. A part of the EM spectrum that is receiving significant attention is the visible band and visible light communications (VLC) is being considered as a way of complementing existing $\mathrm{RF}$ communications channels, especially for indoor communications [4]. The visible band, used in VLC has the advantage that it is easily localised, energy efficient, secure and not susceptible to electromagnetic interference [5].

Since they are already available in most locations the white light LEDs (WLEDs) used to illuminate rooms are an attractive option for use as VLC transmitters [6, 7]. In most WLEDs the white light is generated by a combination of a blue LED and a yellow phosphor [8]. However, the phosphor typically has a slower response, and hence lower bandwidth, than the blue LED [9] and a blue filter is therefore often used at the receiver to increase the bandwidth of the data channel [10]. Once this is done the only way to increase the capacity of the channel is to increase its signal to noise ratio.

One way to increase the signal to noise ratio of a channel is to increase the area that collects light for the photodetector in the receiver. Optical concentrators, such as parabolic concentrators (PC) and compound parabolic concentrators (CPC), are sometimes used to increase the received signal and hence the SNR at the receiver [11]. However, these optical concentrators are limited by etendue and there is therefore a trade-off between gain and field of view [12]. If a wide FOV with a high optical gain are required a fluorescent concentrator (FC) is an attractive alternative [13, 14].

In this paper, the results of a study to investigate the data rate that can be achieved with a fluorescent fibre based concentrator and a white light LED (WLED) are reported. In addition, a method of relating the gain obtained by using a concentrator and the highest achievable OOK data rate is described.

\section{FLUORESCENT CONCENTRATORS FOR VLC/LIFI}

A FC does not only rely upon two processes, reflection and refraction, that conserve etendue [15]. Instead they rely upon a combination of refraction, fluorescence and total internal reflection (TIR) and they can therefore have a wide field of view and a high gain. [16].

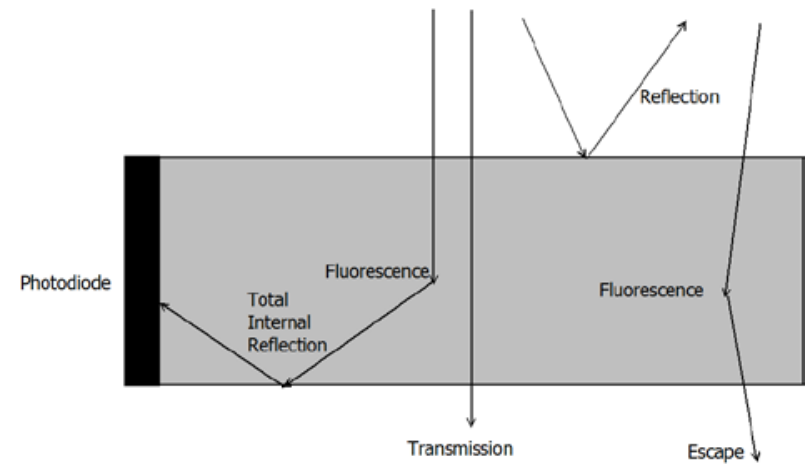

Fig.1 A schematic diagram of the key processes in a fluorescent concentrator.

When a beam of light is incident on a FC, the photons in the beam can undergo a number of processes shown in Fig. 1. In particular, they may be reflected from the FCs top surface or transmitted through the FC. Alternatively, they can be absorbed by the fluorophore in the FC. If the fluorophore has a high quantum yield then most of these absorbed photons will result in another photon that is emitted in a random direction. Although some of these emitted photons will escape the concentrator most will be retained by total internal reflection. Some of these retained photons will be absorbed for a second time and the sequence of events will start again, but those photons that aren't reabsorbed reflect around the concentrator until they reach the photodiode. It is the absorption and re-emission of photons within the FC that mean that, unlike conventional optical concentrators, they are not limited by the etendue [17].

An additional feature of the fluorophores in FCs is that they emit longer wavelengths than they absorb. Typically, silicon photodetectors are designed to be more sensitive to 

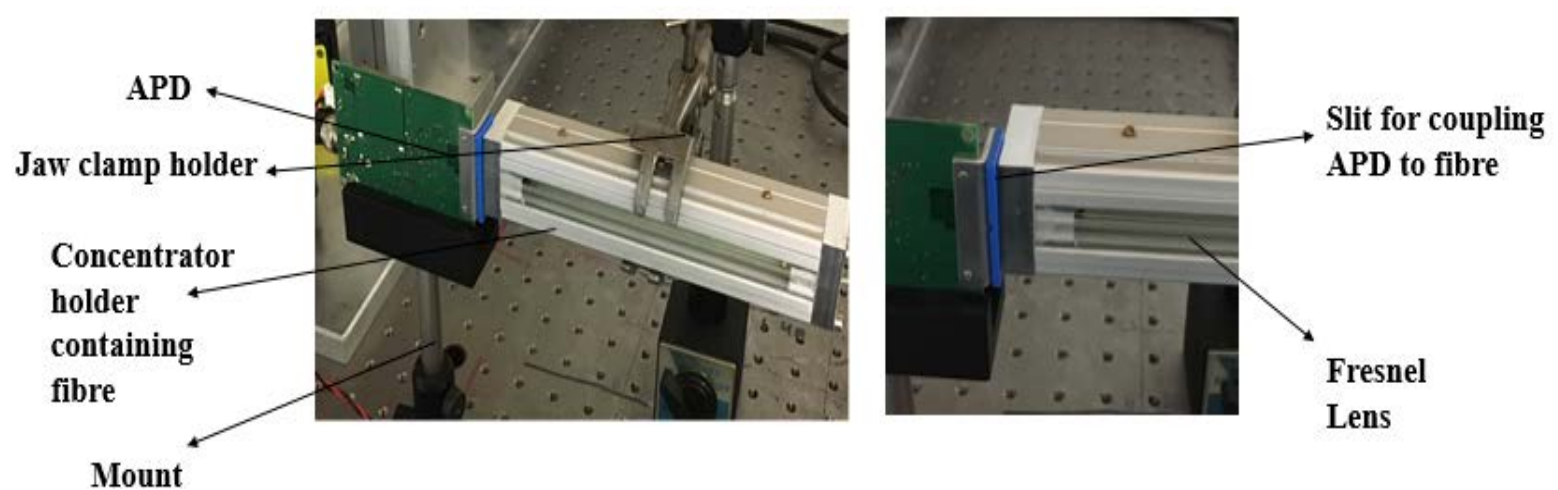

Fig. 2. A photograph on the left shows the APD on the left and the receiver with a Fresnel lens on a holder that ensures the fibre is at the focus of a cylindrical Fresnel lens on the right. The photograph on the right shows the coupling between the fibre and the APD including the blue 3D printed coupler.

longer wavelengths and so this increase in wavelength potentially provides an additional increase in the signal at the receiver. Therefore, the FC gives a receiver a higher sensitivity and larger FOV than a receiver based upon a PD alone or a PD with a conventional optical concentrator. The ability for a receiver containing a FC to surpass the limits set by etendue have been confirmed experimentally [18].

\section{FLUORESCENT FIBRE CONCENTRATOR}

Fluorescent fibres are sold for a variety of applications including for gun or archery sights and for decorative lighting. Coincidentally, and conveniently, they have the same diameter as some fast photodetectors, and so they can be efficiently coupled to these photodetectors. To create an optical concentrator a commercial green emitting fibre has been inserted inside a concentrator holder which has a $13 \mathrm{~cm}$ long plastic Frensel lens at the front and which has one fixture at each end to keep the fibre at the focus of the Fresnel lens. The concentrator holder casing is made up of almunium because it is easy adapted, inexpensive and light weight. For convenience during experiments this concentrator holder was held in a jaw clap and an C1203-07 APD with a bandwidth of $70 \mathrm{MHz}$ was coupled to one end of the fibre. Since the APD has a diameter of $0.5 \mathrm{~mm}$ a 3D printed coupler was used to align the end of the fibre and the APD.

The peak absorption of this fluorescent fibre lies in the blue part of the visible spectrum. Hence the fibre itself acts as a filter that removes the slower yellow phosphor generated portion of the white light before it reachs the
APD. The bandwidth of the fibre was measured in some initial experiments in which a high-speed laser diode (PL450B, $750 \mathrm{MHz}$ BW) was used as the light source. The results of these experiments showed that this fibre has a $3 \mathrm{~dB}$ bandwidth of $24 \mathrm{MHz}$. This indicates that the flourphore in the fibre has a lifetime of $6.6 \mathrm{~ns}$.

\section{CHARACTERISATION OF THE CONCENTRATOR}

In subsequent experiments an OS-PCN-2015-013-A Version 1.2 white LED (WLED) from OSRAM was used as the light source. Based upon the power-current-voltage (LIV) characterstics of this WLED the sum of a $3 \mathrm{~V}_{\mathrm{pp}}$ signal and $3 \mathrm{~V}$ d.c. signal were used to drive the LED through an Agilent 81150A AWG, which has bandwidth of $120 \mathrm{MHz}$. The Hamamatsu C1203-07 APD was then used to detect the signal from the end of the fibre. The output from this APD was connected to a MSO6140B $1 \mathrm{GHz}$ oscilloscope which captured the receiver output. All experiments were performed at the link distance at which the WLED gave 300 Lux.

The advantage of using a WLED to transmit data is that it can simultaneously provide illumination and a data link. However, the $3 \mathrm{~dB}$ bandwidth of the typical WLED is typically less than $10 \mathrm{MHz}$. The bandwidth of the particular WLED used to transmit data was measured experimentally and found to be $2.5 \mathrm{MHz}$, see Fig. 4. Then, to block the light from the yellow phosphor from reaching the APD, a blue filter was introduced in the optical path between the WLED and the APD. As shown in Fig. 4, the bandwidth of the channel was increased to $3.5 \mathrm{MHz}$. Since the fibre only

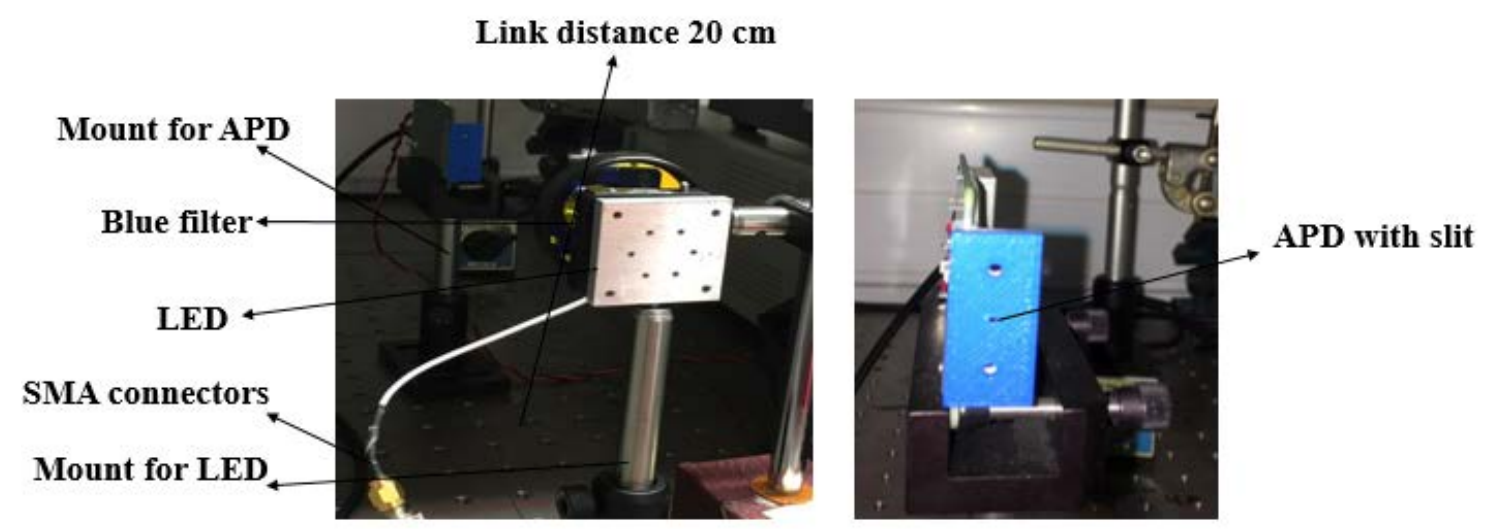

Fig. 3. The experimental arrangement used to determine the bandwidth of the white light LED and to transmit data from the white light LED directly to the APD. The key feature of this experiment is the 3D printed blue cover which reduced the effective area of the APD to the cross sectional area as the fibre. In other experiments this cover was used to align the APD and fibre. 
absorbs blue light it will also prevent the light from the phosphor from reaching the detector. The bandwidth of the link when the fibre is used to collect light from the WLED was therefore measured. The results in Fig. 4 show that when the fibre is used to filter the light from the WLED the effective bandwidth of the channel is $5.5 \mathrm{MHz}$.

The relatively low bandwidth of the WLED transmitter causes inter-symbol interference (ISI) which limits the maximum data rate that can be achieved without equalisation. When the bandwidth of a channel is limited one approach to increasing its capacity is to increase the signal to noise ratio. Since the bandwidth of the fluorescent fibre, $24 \mathrm{MHz}$, is significantly higher than the bandwidth of the transmitter the bandwidth of the fibre is expected to have a negligable effect on the link. The fibre is therefore expected to provide gain in a way that is similar to the gain provided by more conventional optical concentrators.

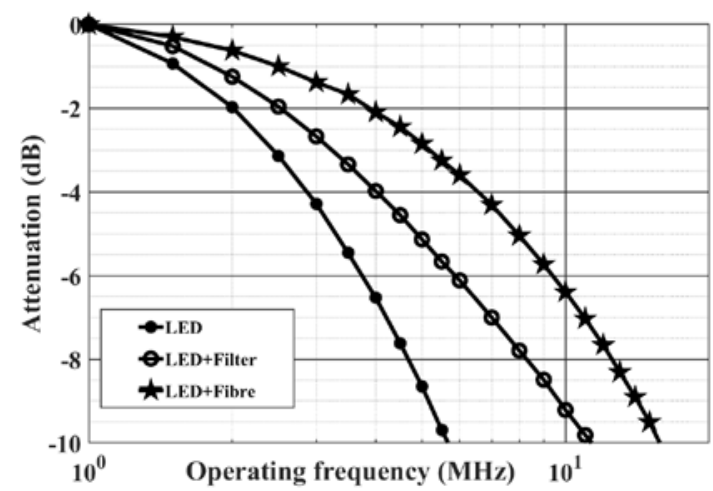

Fig.4.The measured bandwidth of the white LED without a filter, with a blue filter and with the fibre.

\section{COMMUNICATIONS PERFORMANCE}

The performance of the link between the WLED and the reciever that incorporated the fluorescent fibre was tested using the experimental arrangement shown in Fig. 2 and Fig. 3 and the full length of the concentrator. Furthermore, data was transmitted using the bias voltages used in the bandwidth experiments.

For these experiments, a random on-off keying signal was generated in MATLAB. This digital signal was then converted into an analogue signal to drive the LED through the AWG. The white light emitted from the LED was used to illuminate the receiver and an oscilloscope captured the output signal from the APD. This output signal was then processed in MATLAB to extract the bit stream. The extracted bit stream was then compared to the transmitted bit stream so that the bit error rate (BER) could be calculated.

To compare the performance of the link with and without the FC the data communications experimemts were performed in two scenarios i.e. with the APD acting as the reciever and when the FC was coupled to the APD. In these experiments a Hamamatsu C1203-07 APD, with a bandwidth of $70 \mathrm{MHz}$ and an active area of $3 \mathrm{~mm}^{2}$ was used as the detector. However, the diameter of the fibre was only $1.2 \mathrm{~mm}$ and so for this comparison a 3D printed cover with an aperture with a diameter of $1.2 \mathrm{~mm}$ was used to cover the active area of the APD. When the APD was used with the concentrator this 3-D printed aperture was used to avoid any direct leakage of light from the source to the APD and to align the fibre to the APD, Fig. 3.

In the first experiments the APD was placed at the distance from the WLED at which the WLED gave an illuminance of 300 lux. A blue filter was then placed in front of the APD to block the slower phosphor generated component of the white light. Using the APD and OOK it was possible to transmit $17 \mathrm{Mbps}$, without equalization and with a BER of $10^{-3}$. When the APD was replaced by the concentrator and the concentrator was flood illuminating and it was possible more than double the data rate and transmit 38 Mbps.

\section{DATA RATE AS A FUNCTION OF GAIN}

The design of concentrators for future receivers will require several decisions including the fluorophore that should be incorporated in the concentrator, the concentration of this fluorophore and both the shape and size of the concentrator itself. The key characteristics of the fluorophore that will need to be considered include making some of these decisions are the photoluminescence quantum yield (PLQY), the absorption spectrum, the emission spectrum and the lifetime of the fluorophore. However, the PLQY and both the spectra influence the gain of the concentrator whilst its lifetime determines it bandwidth. The designer will therefore need a method of relating the gain and the bandwidth of the concentrator to the maximum achievable data rate. Furthermore, this method will also be useful to receiver designers who are considering incorporating concentrators in their receivers.

The bit error rate (BER) of a communications link depends upon Q. For on-off keying (OOK) Q is the ratio of the worst case separation between the receiver outputs corresponding to logic 0 and logic 1 and to the sum of the root mean square noises in these two levels. The $\mathrm{Q}$ value for a correctable BER of $10^{-3}$ is 3.1.

The separation between the two logic levels depends upon the relationship between the bit rate of the transmitted data and the bandwidth of the channel. In particular, if the bit rate is too high the receiver doesn't have enough time to achieve the maximum separation between levels. In this case extra power is needed to maintain the BER and the transmitted power has to be increased by a factor known as the power penalty, PP. Since the bandwidth of the channel is limited by the single pole response of the LED the power penalty for a bit rate $B$ is

$$
P P=\frac{1}{1-2 \cdot \exp \left(-2 \cdot \pi \cdot B W_{3 d B} / B\right)}
$$

where $B W_{3 d B}$ is the bandwidth of the channel[19].

By increasing the power reaching the photodiode in the receiver a concentrator will allow the bit rate at the required BER to be increased. However, as the data rate is increased the bandwidth of the electronic filter that controls the noise bandwidth in the receiver should also be increased. To take into account the power penalty, the change in noise bandwidth and the $3 \mathrm{~dB}$ frequency of the LED the impact of using concentrators with different gains within a receiver has been calculated numerically. The results of these calculations are shown in Fig 5. 


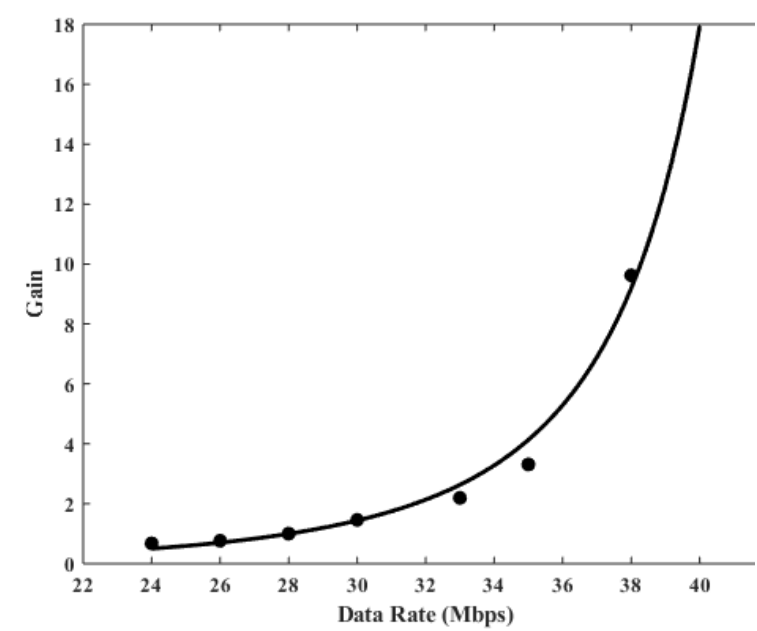

Fig. 5. The expected relationship between increases in the signal gain and data rate when a fibre concentrator is used (solid line) and measured data (dots).

The relationship between gain and data rate has also been explored experimentally. These experiments used the equipment and bias voltages described in sectons IV and V. The gain of the fibre was then varied by shielding different lengths of the concentrator. For each length the peak-topeak voltage when a sine wave was transmitted was measured so that the effective gain of this length of fibre could be determined. Various data rates were then transmitted to the partly covered concentrator to determine the data rate at which a BER of $10^{-3}$ could be achieved with this length of fibre.

The numerical results predicted the impact of adding gain into a link that has already achieved the required BER. This meant that to compare the experimental results to the numerical predictions one of the data points had to be used as the reference point and associated with a gain of 1 . The effective gains of all the other data points were then calculated using the ratio of the peak-to-peak responses to a transmitted sine wave. The resulting effective gains and corresponding data rates are shown as points in Fig. 5. This figure also shows the results of the numerical predictions. A comparison between the experimental results and the numerical predictions shows that the suggested method is accurate enough to be used to both design concentrators and to predict the impact of adding a concentrator into an existing link.

\section{CONCLUSION AND FUTURE WORK}

Results have been presented which show that a commercially available fluorescent fibre can be used to create a fluorescent concentrator for a VLC reciever. When this concentrator is used with a white light LED its absorption properties filter out the faster blue component of the white light. This increases the effective bandwidth of the link from $2.5 \mathrm{MHz}$ to $5.5 \mathrm{MHz}$. In addition the use of the fibre more than doubles the data rate that can be achieved when the white LED is providing an illumination of 300 lux.

In addition to these results a method has been described which can be used to predict the relationship between gain, bandwidth and maximum data rate. It is anticipated that this method will be used to select the optimum flourophore for incorporation into concentrators and to predict the impact of a concentrator on the performance of a VLC link.

Further work is needed to determine the impact of concentrators on links that also employ equalisation.

\section{REFERENCES}

[1] A. R. Ndjiongue and H. C. Ferreira, "Visible Light Communications (VLC ) Technology,” no. October 2017, 2015.

[2] M. Kavehrad, "Optical wireless applications: a solution to ease the wireless airwaves spectrum crunch,” vol. 8645, p. 86450G-1, 2013

[3] K. Pretz, “Overcoming Spectrum Scarcity,” The Institute: IEEE, Aug2012.

[4] S. Hranilovic, Wireless Optical Communication Systems. Springer, 2005.

[5] D. O’Brien and L. Zeng, "Visible light communications: Challenges and possibilities,” PIMRC, IEEE 19th International Symposium on, 2008

[6] Medina, C., Zambrano, M. and Navarro, K., 2015. Led based visible light communication: Technology, applications and challenges-a survey. International Journal of Advances in Engineering \& Technology, 8(4), p.482.

[7] C. Liao, Y. Chang, and C. Ho, "Light-Emitting Diodes for Visible Light Communication,” no. Vlc, pp. 665-667, 2015.

[8] O'Brien, D.C., 2011, October. Visible light communications: challenges and potential. In Photonics Conference (PHO), 2011 IEEE (pp. 365-366). IEEE.

[9] Chun, H., Rajbhandari, S., Faulkner, G., Tsonev, D., Xie, E., McKendry, J.J.D., Gu, E., Dawson, M.D., O'Brien, D.C. and Haas, H., 2016. LED based wavelength division multiplexed $10 \mathrm{~Gb} / \mathrm{s}$ visible light communications. Journal of lightwave technology, 34(13), pp.3047-3052.

[10] Chun, H., Rajbhandari, S., Faulkner, G. and O'Brien, D., 2014, September. Effectiveness of blue-filtering in WLED based indoor visible light communication. In Optical Wireless Communications (IWOW), 2014 3rd International Workshop in(pp. 60-64). IEEE.

[11] R. Winston, "Dielectric compound parabolic concentrators.," Appl. Opt., vol. 15, no. 2, pp. 291-292, 1976.

[12] R. Winston, W. T. Welford, J. C. Miñano, and P. Benítez, Nonimaging Optics. 2005.

[13] Collins, S., O’Brien, D.C. and Watt, A., 2014. High gain, wide field of view concentrator for optical communications. Optics letters, 39(7), pp.1756-1759.

[14] Peyronel, T., Quirk, K.J., Wang, S.C. and Tiecke, T.G., 2016. Luminescent detector for free-space optical communication. Optica, 3(7), pp.787-792.

[15] Manousiadis, P.P., Rajbhandari, S., Mulyawan, R., Vithanage, D.A., Chun, H., Faulkner, G., O’Brien, D.C., Turnbull, G.A., Collins, S. and Samuel, I.D., 2016. Wide field-of-view fluorescent antenna for visible light communications beyond the étendue limit. Optica, 3(7), pp.702-706.

[16] Mulyawan, R., Chun, H., Gomez, A., Rajbhandari, S., Faulkner, G., Manousiadis, P.P., Vithanage, D.A., Turnbull, G.A., Samuel, I.D., Collins, S. and O'Brien, D., 2017. MIMO visible light communications using a wide field-of-view fluorescent concentrator. IEEE Photonics Technology Letters, 29(3), pp.306-309.

[17] Collins, S., Mulyawan, R., Rajbhandari, S., Chu, H., Faulkner, G.E., O'Brien, D.C., Manousiadis, P.P., Vithanage, D.A., Turnbull, G.A. and Samuel, I.D.W., 2015, July. A simple wide field of view concentrator for free space visible light communications. In Summer Topicals Meeting Series (SUM), 2015 (pp. 43-44). IEEE.

[18] Mulyawan, R., Gomez, A., Chun, H., Rajbhandari, S., Manousiadis, P.P., Vithanage, D.A., Faulkner, G., Turnbull, G.A., Samuel, I.D., Collins, S. and O'Brien, D., 2017, January. A comparative study of optical concentrators for visible light communications. In Broadband Access Communication Technologies XI (Vol. 10128, p. 101280L).

[19] Eduard Sackinger "Analysis and Design of Transimpedance Amplifiers for Optical Receivers” Wiley (2018). 